\title{
INCIDENCIA DE NEOPLASIA INTRAEPITELIAL CERVICAL EN PACIENTES QUE ACUDEN A LA CLÍNICA DE PATOLOGÍA DE CÉRVIX EN UN HOSPITAL DE SANTO DOMINGO, REPÚBLICA DOMINICANA, DURANTE EL PERÍODO ENERO-DICIEMBRE 2014 \\ Incidence rates of cervical intraepithelial neoplasia among patients that attend a Cervical Pathology Clinic in Santo Domingo, Dominican Republic, from January to December 2014
}

\section{Nicole Marie Barreto Rojasa , Estefanía Henríquez Luthjeb, Génesis Edith Alcántara Espejoc, Laura Rebeca Esquea Pagés ${ }^{\text {y }}$ Daniel Richardson ${ }^{e}$}

Recibido: 6 de abril, 2019 • Aprobado: 20 de julio, 2019

Cómo citar: Barreto Rojas NM, Henríquez Luthje E, Alcántara Espejo GE, Esquea Pagés LR, Richardson D. Incidencia de neoplasia intraepitelial cervical en pacientes que acuden a la Clínica de Patología de Cérvix en un hospital de Santo Domingo, República Dominicana, durante el período enero-diciembre 2014. cysa [Internet]. 23 de noviembre de 2019 [citado 25 de noviembre de 2019];3(3):51-8. Disponible en: https://revistas.intec.edu.do/index.php/cisa/article/view/1539

\section{Resumen}

Introducción: las neoplasias intraepiteliales cervicales (NIC) son lesiones precursoras del cáncer de cérvix. Aproximadamente cada ańo se registran 527,600 nuevos casos, posicionándolo como el segundo cáncer más frecuente y la segunda causa mundial de muerte por cáncer en la mujer.

Objetivo: determinar la incidencia de NIC en pacientes que acudieron a una Clínica de Patología de Cérvix de Santo Domingo, República Dominicana.

Material y métodos: estudio retrospectivo, descriptivo y transversal en el cual se revisaron las historias médicas de 144 pacientes en riesgo de ser diagnosticadas con NIC durante el periodo.

Resultados: la incidencia de NIC fue de $40.97 \%$ (N=144). La NIC I fue el diagnóstico más frecuente con un $93.22 \%$ $(\mathrm{N}=59)$, seguido $6.78 \%$ con NIC II $(\mathrm{N}=59)$. Hubo una mayor frecuencia de NIC en las edades entre los 30 y 59 años $(72.88 \%, \mathrm{~N}=59) .62 .71 \%(\mathrm{~N}=59)$ presentó infección

\footnotetext{
a Estudiantes de Medicina del Instituto Tecnológico de Santo Domingo (INTEC). Correo-e: nicolembarreto@hotmail.com

b Estudiantes de Medicina del Instituto Tecnológico de Santo Domingo (INTEC). Correo-e: ehenriquezluthje@gmail.com
}

\begin{abstract}
Introduction: Cervical intraepithelial neoplasia (CIN) are precursor lesions of cervical cancer. Every year there are approximately 527,600 new cases, ranking cervical cancer as the second most frequent cause of cancer and the second cause of cancer deaths in women worldwide.

Objective: Determine the incidence of CIN among patients that attend a cervical pathology clinic in Santo Domingo.

Methods: Retrospective, descriptive and cross-sectional study in which 144 medical histories of patients in risk of being diagnosed with CIN during the study period were studied.

Results: The incidence of CIN was $40.97 \%(\mathrm{~N}=144)$. The majority of cases were diagnosed with CIN I, for a total of $93.22 \%(\mathrm{~N}=59)$, followed by $6.78 \%$ diagnosed with CIN II ( $\mathrm{N}=59)$. A higher frequency of CIN was found between the ages of 30 and $59(72.88 \%, \mathrm{~N}=59) .62 .71 \%$

\footnotetext{
c Estudiantes de Medicina del Instituto Tecnológico de Santo Domingo (INTEC). Correo-e: g.alcantaraespejo@hotmail.com

d Estudiantes de Medicina del Instituto Tecnológico de Santo Domingo (INTEC). Correo-e: laura3esquea@gmail.com

e Médico Gineco-Obstetra Oncólogo
} 
por virus del papiloma humano $(\mathrm{VPH})$. La paridad en el $42.86 \%(\mathrm{~N}=35)$ fue mayor de cuatro. $50 \%(\mathrm{~N}=16)$ reportó como hábito tóxico el tabaquismo.

Conclusiones: el diagnóstico de NIC predomina entre los 30 y 59 ańos, con una mayor frecuencia de NIC I. La infección por $\mathrm{VPH}$, la paridad y el tabaquismo son factores que pudieran tener influencia sobre el desarrollo de la NIC.

Palabras clave: cáncer de cérvix; neoplasias uterinas; displasia del cuello del útero; virus del papiloma humano; cáncer ginecológico.

\section{Introducción}

Se conoce como neoplasia intraepitelial cervical (NIC) a las anomalías de desarrollo en las cuales el tejido epitelial pierde su estructura. Se presentan además alteraciones en la morfología de los componentes celulares de este epitelio ${ }^{1}$. En sus primeras etapas las lesiones son totalmente curables, mas de no hacer un diagnóstico temprano estas lesiones pudiesen ser precursoras del cáncer de cuello uterino $(\mathrm{CCU})^{2}$.

El CCU constituye el segundo cáncer más común y la segunda causa de muerte por cáncer en la mujer a nivel mundial, con aproximadamente 527,600 nuevos casos diagnosticados y 265,700 defunciones anuales $^{4-8}$. En la República Dominicana, el CCU oscila entre los primeros dos lugares en los cánceres que afectan a las mujeres ${ }^{9}$, mientras que en países desarrollados este cáncer se coloca en puestos más elevados que el quinto ${ }^{10}$.

El objetivo de esta investigación fue determinar la incidencia de NIC en pacientes que acuden a la Clínica de Patología de Cérvix en un Hospital de Santo Domingo, República Dominicana, durante el período enero-diciembre 2014. Esto es importante porque alrededor del $90 \%$ de los diagnósticos anuales a nivel mundial de CCU ocurren en países en vías de desarrollo, como es la República Dominicana ${ }^{7}$.
$(\mathrm{N}=59)$ were infected with the human papillomavirus (HPV). 42.86\% (N=35) had more than four children. $50 \%(\mathrm{~N}=16)$ were smokers.

Conclusions: $\mathrm{CIN}$ are most frequently diagnosed in the ages between 30 and 59 years, being CIN I the most prevalent diagnosis. HPV infection, parity and smoking are factors that might influence the development of CIN.

Keywords: Cervical cancer; uterine neoplasms; cervical dysplasia; human papillomavirus; gynecologic cancer.

Las NIC representan la etapa predecesora del CCU, lo que manifiesta la vital importancia de su estudio si se desea disminuir la incidencia de este cáncer, y las implicaciones médicas, económicas, sociales y psicológicas asociadas al mismo ${ }^{3}$.

\section{Clasificación}

Actualmente, las lesiones son clasificadas por patólogos a través del uso de microscopios, con la finalidad de evaluar muestras de tejido basándose en características como el número de células displásicas. La NIC se caracteriza por la manifestación de atipia y el pleomorfismo en las células escamosas del cérvix.

Basado en el grado de pleomorfismo y atipia citológica, las NIC tradicionalmente se clasifican de la siguiente manera: NIC I (displasia leve) o de bajo grado, NIC II (displasia moderada) y NIC III (en un espectro que incluye la displasia severa y el carcinoma in situ, ambas referidas como lesiones escamosas intraepiteliales de alto grado). Mientras que la NIC I muestra células atípicas confinadas al tercio basal del epitelio del cérvix, la NIC II se asocia a los primeros dos tercios, y la NIC III al reemplazo total del epitelio nativo ${ }^{11}$.

\section{Sintomas y Caracteristicas Clínicas}

La presencia de la NIC no manifiesta síntomas específicos o características clínicas. No obstante, 
estas lesiones pueden tornarse de color blanco posterior a la aplicación de ácido acético al 3-5\% en la zona de transición del cérvix (la unión entre el epitelio escamoso y el epitelio columnar), y ser también yodonegativas tras aplicar lugol, debido a que el epitelio de la NIC suele contener escaso, o ningún glucógeno ${ }^{12}$.

\section{Etiología}

La infección persistente por el papillomavirus de alto riesgo, específicamente por los serotipos 16, 18, 31, 33, 35 y 39, entre otros, del virus del papiloma humano (VPH) se asocian fuertemente a la NIC y al cáncer invasor ${ }^{12}$. Su incidencia se incrementa con una edad temprana de inicio de relaciones sexuales, las múltiples parejas sexuales, y una pareja masculina con múltiples parejas sexuales previas. Otros factores de riesgo definidos son el tabaquismo y la inmunodeficiencia ${ }^{13}$.

\section{Diagnóstico}

El diagnóstico presuntivo de la NIC se efectúa a través de citología cervical, colposcopia, y conización, y el definitivo a través de la biopsia histopatológica ${ }^{14}$. Se inicia a través de la citología cervical, como técnica de pesquisa, con el propósito de detectar la presencia de células anormales. La prevención del CCU se ha centrado en el tamizaje de las pacientes, tomando la muestra de la zona de transición del cérvix. Pacientes con resultados anormales son sometidas a la evaluación colposcópica, con fines diagnósticos. Si se observan imágenes atípicas, se toma una biopsia de la lesión para ser sometida a un estudio histopatológico?.

\section{Tratamiento}

En pacientes con NIC I, se efectúa un seguimiento periódico a través de la citología cervical, debido a que estas lesiones suelen remitir espontáneamente. En contraste, en pacientes con lesiones de NIC II y III, el tratamiento recomendado es la escisión, para detener la progresión hacia un carcinoma ${ }^{11}$.
Actualmente, se encuentran disponibles además la crioterapia, el procedimiento de extirpación electroquirúrgico de lazo, el procedimiento de extirpación electroquirúrgico de lazo en la zona de transformación, conización en frío, vaporización láser, coagulación fría e histerectomía ${ }^{15}$.

\section{Materiales y Métodos}

Este estudio fue de carácter retrospectivo, debido a que se indagó en datos pertenecientes al pasado período enero-diciembre 2014, de fuentes secundarias. Descriptivo, pues buscaba determinar cuál era la situación de las variables que se estudiaron en la población: la presencia e incidencia de la patología, en quiénes, dónde y cuándo se presentaba la misma; además, se detallaron los resultados tal y como se presentaron; y de corte transversal, dado que se estudiaron las variables y sus respectivas dimensiones e indicadores simultáneamente en un determinado momento, sin dar continuidad de seguimiento a las mismas en el eje cronológico.

Los datos fueron obtenidos mediante el empleo de un cuestionario impreso como instrumento de recolección de datos, elaborado por las autoras y el asesor de la presente investigación. Este cuestionario se aplicó sobre las historias clínicas de las pacientes que acudieron a la Clínica de Patología de Cérvix de un hospital de Santo Domingo, República Dominicana, y que fueron diagnosticadas con NIC durante el período enero-diciembre 2014. Las variables del cuestionario incluyen la edad, el método diagnóstico, la presencia de infección por el VPH, los antecedentes ginecobstétricos y los antecedentes tóxicos.

El universo estudiado abarcó a todas las pacientes diagnosticadas con NIC en la Clínica de Patología de Cérvix en un hospital de Santo Domingo. Asimismo, la población estudiada estuvo comprendida por las 59 pacientes diagnosticadas con NIC tratadas durante el período enero-diciembre en la Clínica de Patología de Cérvix en un hospital de Santo Domingo. El nombre 
del mismo no fue especificado debido a la confidencialidad requerida, y durante el transcurso del estudio se llevó a cabo todo lo necesario para mantener el anonimato de las identidades de las pacientes.

El criterio de inclusión de la presente investigación comprendió: ser un caso incidente de NIC I, NIC II o NIC III, durante el período enero-diciembre 2014. Los criterios de exclusión comprenden a las pacientes que no pertenecían al período establecido, las que no desarrollaron NIC, o las que llegaron a la fase de Carcinoma In Situ.

Los datos obtenidos fueron insertados en una base de datos elaborada a través del programa Microsoft Excel para Mac 2011 versión 14.4.7.

\section{Resultados}

Gráfica 1. Incidencia de neoplasia intraepitelial cervical. $(\mathrm{N}=144)$

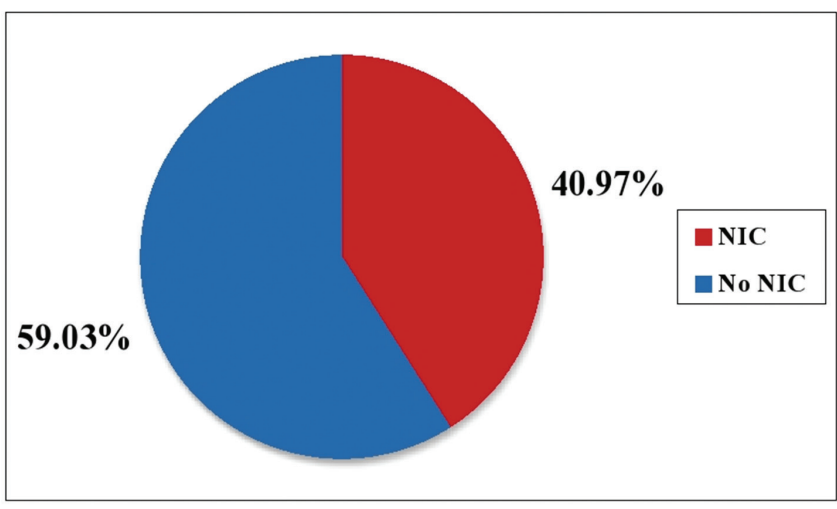

Fuente: elaboración propia.

Esta gráfica muestra el hallazgo de NIC, con la finalidad de determinar la incidencia. En la misma se presenta el porcentaje total de nuevos casos de NIC frente al porcentaje de los casos que no presentan NIC, dentro de un número de 144 pacientes que se encontraban en riesgo de desarrollar la enfermedad al inicio del período de estudio. Se observa que 59 pacientes fueron diagnosticadas con NIC, lo que representa el $40.97 \%$ de estos casos en riesgo. De igual forma, el $59.03 \%$, es decir, 85 pacientes, no fueron diagnosticadas con NIC a pesar de pertenecer al grupo de pacientes en riesgo.

Tabla 1. Distribución según grupos etarios. ( $\mathrm{N}=59)$

\begin{tabular}{|l|c|c|c|c|}
\hline \multirow{2}{*}{$\begin{array}{l}\text { Grupos } \\
\text { Etarios } \\
\text { Años) }\end{array}$} & \multicolumn{2}{|c|}{ Resultado Diagnóstico } & TOTAL \\
\cline { 2 - 5 } & NIC I & NIC II & NIC III & Número - (\%) \\
\hline$\leq \mathbf{1 9}$ & $0(0 \%)$ & $0(0 \%)$ & $0(0 \%)$ & $0(0 \%)$ \\
\hline $\mathbf{2 0 - 2 9}$ & $6(10.91 \%)$ & $0(0 \%)$ & $0(0 \%)$ & $6(10.17 \%)$ \\
\hline $\mathbf{3 0 - 3 9}$ & $10(18.18 \%)$ & $0(0 \%)$ & $0(0 \%)$ & $10(16.95 \%)$ \\
\hline $\mathbf{4 0 - 4 9}$ & $14(25.45 \%)$ & $1(25 \%)$ & $0(0 \%)$ & $15(25.42 \%)$ \\
\hline $\mathbf{5 0 - 5 9}$ & $18(32.73 \%)$ & $0(0 \%)$ & $0(0 \%)$ & $18(30.51 \%)$ \\
\hline$\geq \mathbf{6 0}$ & $7(12.73 \%)$ & $3(75 \%)$ & $0(0 \%)$ & $10(16.95 \%)$ \\
\hline TOTAL & $55(93.22 \%)$ & $4(6.78 \%)$ & $0(0 \%)$ & $59(100 \%)$ \\
\hline
\end{tabular}

Fuente: elaboración propia.

En esta tabla se presenta la distribución del diagnóstico de las pacientes según los grupos etarios, precedida de la distribución total de las pacientes con NIC según edades. Se puede apreciar que hubo una mayor incidencia de NIC I, con 55 casos para un $93.22 \%$. En el caso de NIC II, se registraron 4 casos, lo que equivale a un $6.78 \%$ de la población. No se hallaron casos de NIC III.

En la distribución de todas las NIC según la edad (columna Número -[\%]), se observa que 18 pacientes pertenecen al grupo etario entre los 50-59 años, lo que representa un $30.51 \%$ de la población total; 15 pertenecen al grupo etario entre 40-49 años, lo que equivale a un $25.42 \%$ de la población; 10 pertenecen al grupo etario entre 30-39, lo que representa un $16.96 \%$ de la población, al igual que el grupo etario mayor o igual a 60 ańos; 6 pacientes pertenecen al grupo etario entre 20-29 años, lo que equivale a un $10.17 \%$ de la población total; mientras 
que no se presentó ningún caso en el grupo etario menor o igual a los 19 ańos.

Gráfica 2. Infección por virus del papiloma humano (VPH). ( $\mathrm{N}=59)$

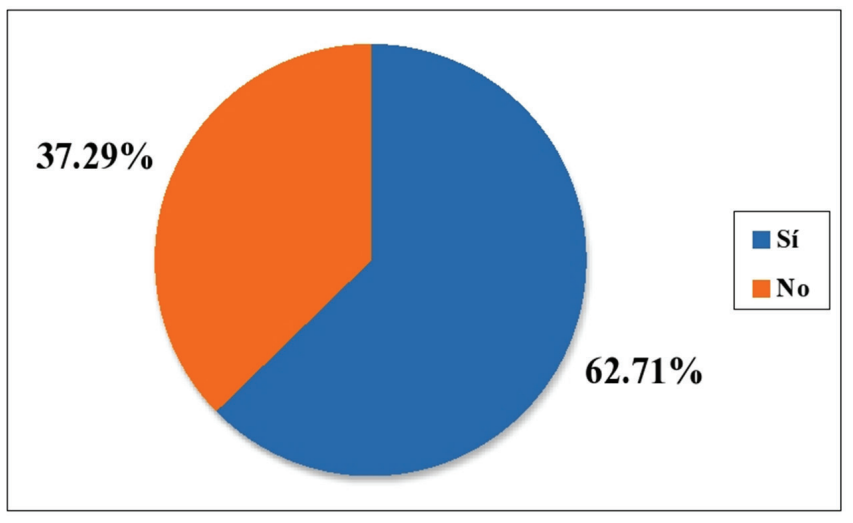

Fuente: elaboración propia.

Esta gráfica muestra la distribución de las pacientes con NIC a partir de la presencia de infección por el VPH. En la misma se evidencia el porcentaje total de la presencia o no del VPH en la población de estudio, compuesta por 59 mujeres diagnosticadas con NIC. La gráfica muestra que 37 mujeres se encontraron infectadas por el VPH, para un total del $62.71 \%$ de la población total; mientras que el $37.29 \%$ de la población no se encontró infectada, es decir, 22 casos.

Gráfica 3. Paridad. $(\mathrm{N}=35)$

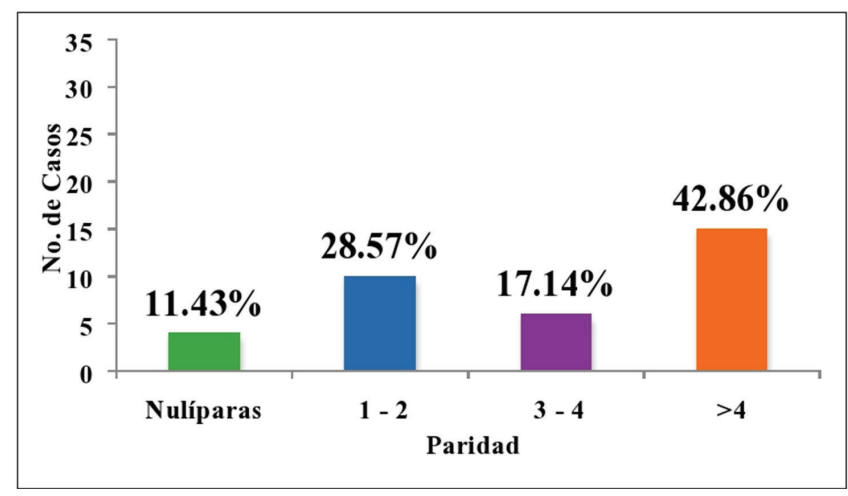

Fuente: elaboración propia.
Esta gráfica muestra la distribución de las pacientes con NIC según la paridad. En la misma se presenta el porcentaje total de número de partos que haya tenido la paciente diagnosticada con NIC, utilizando como población de estudio a 35 pacientes. A pesar de que se registró una cifra de 59 pacientes diagnosticadas con NIC, solo 35 historias clínicas registraron la paridad, tomando esta cifra para la evaluación para la presente variable. Se muestra que 15 mujeres tuvieron más de 4 partos, representando un $42.86 \%$ de la población; 10 casos presentaron de 1 a 2 partos, lo que equivale al $28.57 \%$ de la población total; 6 pacientes tuvieron de 3 a 4 partos, para un total del $17.14 \%$ de la población; mientras que 4 de las pacientes fueron nulíparas, lo que equivale al $11.43 \%$ de la población total.

Gráfica 4. Tabaquismo. $(\mathrm{N}=16)$

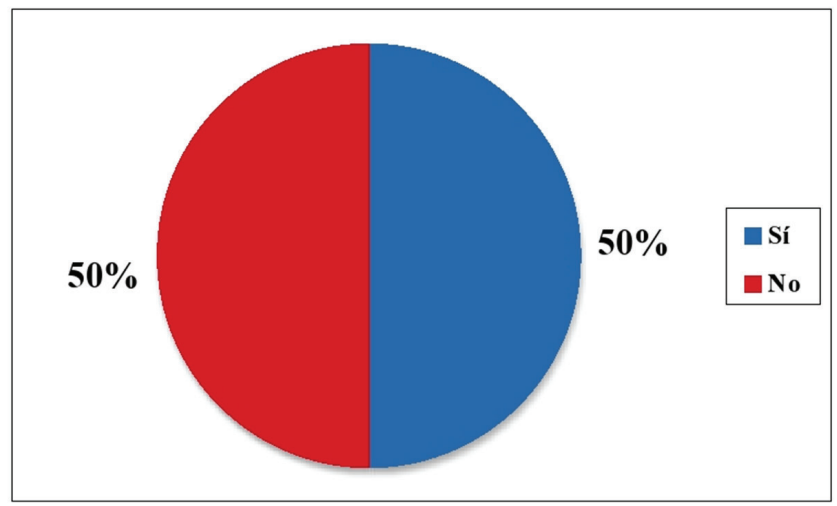

Fuente: elaboración propia.

En esta gráfica se muestra el hábito del tabaquismo en pacientes con NIC. En la misma se evidencia el porcentaje total de pacientes que señalan tener o no el hábito de fumar, en la población de estudio compuesta por 16 pacientes diagnosticadas con NIC en la Clínica de Patología de Cérvix. De la cifra total de 59 historias clínicas con el diagnóstico de NIC, solo en 16 historias se llenó la casilla correspondiente al tabaquismo, por lo que las demás no fueron valorables para esta variable. De una población de 16 pacientes, se observa que 8 pacientes sí poseen 
el hábito de fumar, lo que representa el $50 \%$ de la población, y el otro $50 \%$ de la población no posee este hábito, para un total de 8 casos.

\section{Discusión y Conclusiones}

En el presente estudio, la incidencia de NIC en pacientes que acudieron a la Clínica de Patología de Cérvix en un hospital de Santo Domingo fue de 40.97 \% (gráfica 1). Una investigación realizada en la Consulta Provincial de Patología de Cuello Uterino de un Policlínico Universitario en Cuba demostró una cifra similar, con una incidencia de $51.3 \%$ en el año $2010^{16}$.

Según un estudio realizado en Cuba en el año 2012, por los doctores Lau Serrano y Fajardo Tornés, junto a Sánchez Alarcón (MSc.) y Millán Vega (MSc.), en los resultados citológicos anormales predominó la NIC I, seguido por la NIC II, y en último lugar la NIC III ${ }^{6}$. En la República Dominicana se han registrado estadísticas similares, según una publicación en la Revista Médica Dominicana de mayo-agosto del $2006^{17}$. En el grupo de 59 resultados de citologías cervicales comprendidas durante el período estudiado por la presente, se evidenció, al igual que en el estudio previamente mencionado, el predominio del diagnóstico de NIC I, con un $93.22 \%$ de la población, seguido por la NIC II, representado por un $6.78 \%$ de las pacientes (tabla 1 ).

Un tamizaje realizado en Perú bajo la supervisión de la Organización Panamericana de la Salud (OPS) en el 2005 mostró que el grupo etario comprendido entre los 35 y 50 ańos es el más propenso al desarrollo del CCU, el cual es el sucesor de la NIC ${ }^{3}$. Asimismo, las Normas de Prevención, Diagnóstico Temprano y Tratamiento de Cáncer Cérvico-Uterino de la República Dominicana establecen que el grupo de mayor riesgo de CCU es aquel entre los 35 y 64 años ${ }^{9}$. Esta investigación demostró que dentro de las edades comprendidas entre los 30 y los 59 años se observa una frecuencia de NIC del $72.88 \%$, concluyendo que es la población en mayor riesgo de desarrollar $\mathrm{CCU}$, hecho que reafirma los mencionados estudios (tabla 1).

Aproximadamente, el $80 \%$ de las lesiones epiteliales de bajo grado, y el $100 \%$ de las lesiones epiteliales de alto grado, están asociadas en especial a los serotipos de VPH de alto riesgo ${ }^{3}$. Esto se debe a que es el oncovirus causante de los cambios epiteliales en la zona de transición. ${ }^{18,19}$ Asimismo, estudios moleculares, clínicos y epidemiológicos han evidenciado inequívocamente la relación causal entre el VPH y el $\mathrm{CCU}^{4}$. Un estudio afirma la presencia del VPH en más de la mitad de los resultados citológicos de las pacientes afectadas por la $\mathrm{NIC}^{6}$, dato corroborado por el presente estudio, en el cual el $63 \%$ de las pacientes presentó infección por VPH (gráfica 2).

Un hallazgo de notable importancia fue la paridad, donde el $42.86 \%$ de las pacientes afirmó haber tenido más de cuatro partos (gráfica 3). De acuerdo a una investigación publicada en el National Journal of Community Medicine mostró que un $48.75 \%$ de afectadas habían tenido cuatro o más partos (20), corroborando con la presente investigación. Esta información reafirma las estadísticas que sugieren que las mujeres con tres o cuatro embarazos de término tienen un riesgo 2.5 veces más alto de padecimiento de CCU que las mujeres nulíparas, debido a los traumatismos del parto ${ }^{3,6,10,18}$.

Por otra parte, se ha demostrado que el hábito del tabaquismo, sobre todo desde temprana edad, tiene un efecto nocivo en las secreciones vaginales, específicamente en el epitelio mucíparo del canal endocervical en donde se reserva el VPH. Según una investigación realizada en la Universidad Nacional Autónoma de México, se observó que un $55 \%$ de las pacientes reportó el hábito del tabaquismo, mientras que el $45 \%$ reportó no ser fumadora ${ }^{1}$. La presente investigación halló una proporción similar, 
en la cual el $50 \%$ reportó fumar, mientras que el otro $50 \%$ no fuma (gráfica 4 ).

Se resalta la importancia del diagnóstico temprano, por lo que se recomienda la realización de citologías cervicales periódicas, con la finalidad de detectar casos de lesiones u otros hallazgos. Se recomienda, además, que a todas las pacientes con resultados citológicos anormales se les garantice tener acceso a una consulta especializada para atenderse, donde se cuente tanto con el equipamiento básico como con personal capacitado, y los materiales necesarios para la realización de la histopatología correspondiente. Con la prevención y el tratamiento adecuado, se reduciría la progresión de este tipo de lesiones, lo que consecuentemente disminuiría la incidencia de CCU, y, por ende, aumentaría la calidad de vida de la mujer dominicana.

\section{Bibliografía}

1. Alaniz Sánchez A, Flores Grimaldo J, Salazar Alarcón CE. Factores de riesgo para el desarrollo de la displasia leve cervical. Rev Fac Med UNAM. 2009;52(2): 69-72.

2. Barbón A. Análisis de Algunos Factores de Riesgo de Lesiones Premalignas de Cuello Uterino en un Área de Salud. Rev Haban Cienc Méd [en línea] 2009 [fecha de acceso 10 de marzo de 2015];8(4). Disponible en: http://scielo.sld.cu/ pdf/rhcm/v8n4/rhcm05409.pdf

3. Dávila Gómez H, García Valdés A, Álvarez Castillo F, Castillo Blanco Y, Imengana Fonte L, Matos Rodríguez Z. Neoplasia intraepitelial de cuello uterino en un área de salud de la Isla de la Juventud, Cuba: 1999-2008. Rev Chil Obstet Ginecol. 2011 76(4): 236-43.

4. Camacho Rodríguez D, Reyes Ríos LA, González Ruiz GE. Lesiones Neoplásicas De Cuello Uterino En Mujeres De Una Universidad Colombiana. Hacia la Promoción de la Salud. 2013;18(1): $12-25$.
5. Tao L, Han L, Li X, Gao Q, Pan L, Wu L, et al. Prevalence and risk factors for cervical neoplasia: a cervical cancer screening program in Beijing. BMC Public Health [en línea] 2014 [fecha de acceso 10 de marzo de 2015]. Disponible en: http://www.ncbi.nlm.nih.gov/pmc/ articles/PMC4256817/

6. Lau Serrano D, Millán Vega MM, Fajardo Tornés Y, Sánchez Alarcón C. Lesiones preinvasivas del cuello uterino. Rev Cubana Obstet Ginecol. 2012;38(3): 366-77.

7. Torre LA, Bray F, Siegel RL, Ferlay J, Lortet-Tieulent J, Jemal A. Global cancer statistics, 2012. CA Cancer J Clin. 2015;65(2): 87-108.

8. Treviño-Salinas EM, Vidal-Gutiérrez O, Saldívar-Rodríguez D, Luna-Rivera MJ, Castillo-Sáenz L, Núñez-Alvar RD et al. Evaluación de la incidencia de displasia cervical en embarazadas en un Hospital Universitario. Medicina Universitaria. 2010;12(49): 213-5.

9. Normas de Prevención, Diagnóstico Temprano y Tratamiento de Cáncer Cérvico-Uterino. (2007). Series de Normas Nacionales No. 13 [en línea] (2), pp. 19-20. Disponible en: https:// www.google.com/url? sa =t\&rct=j\&q=\&esr$\mathrm{c}=\mathrm{s} \&$ source $=$ web \& $\mathrm{cd}=1 \& \mathrm{ved}=2 \mathrm{ahUKEwi}-$ 6zPGwvYHlAhUDi6wKH c B J B pUFjAAegQIAxAC\&url=http\%3A\%2 F\% 2 Fsaludysociedadcivil.org.do $\% 2$ Findex. php $\% 2$ Fdocumentos $\% 2$ F $47 \% 2$ FNacional $\%$ 2F126\%2FNormas_de_Prevencio_n_Diagno_stico_Temprano_y_Tratamiento_de_Ca_ ncer_Ce_rvico-Uterino_2007.pdf\&usg=AOvVaw0-e5FffknKjOTuEImldxLp

10. Gupta K, Malik NP, Sharma VK, Verma N, Gupta A. Prevalence of cervical dysplasia in western Uttar Pradesh. J Cytol. 2013;30(4): 257-62. 
11. Fabrizii M, Moinfar F, Jelinek HF, Karperien A, Ahammer H. Fractal Analysis of Cervical Intraepitelial Neoplasia. Plos One [en línea] 2014 [fecha de acceso 10 de marzo de 2015];9(10). Disponible en: http://www.ncbi. nlm.nih.gov/pmc/articles/PMC4193757/pdf/ pone.0108457.pdf

12. Sellors JW, Sankaranarayanan R. La colposcopia y el tratamiento de la neoplasia intraepitelial cervical: Manual para principiantes. Lyon: Centro Internacional de Investigaciones sobre el Cáncer; 2005: 13-9.

13. Kumar V, Abbas AK, Fausto N, Mitchell RN. Aparato Genital Femenino y Mama. En: Robbins, Patología Básica. Barcelona: Elsevier España, 2008. p. 711-50.

14. Féliz L, Sánchez M, Santana L, Santana M, Matos M, Francisco C, et al. Correlación Diagnóstica entre Citología, Colposcopía y Biopsia en la Neoplasia Intraepitelial Cervical, Hospital de la Mujer Dominicana. Rev Med Dom. 2011;72(3): 107-10.

15. World Health Organization. Use of cryotherapy for cervical intraepithelial neoplasia. Ginebra: OMS; [en línea] 2011: 1 [fecha de acceso 7 de marzo de 2015]. Disponible en: https:// www.who.int/reproductivehealth/publications/ cancers/ 9789241502856/en/
16. Reyes Zuñiga YI, Sanabria Negrín JG, Marrero Fernandez R. Incidencia y mortalidad por cáncer cervicouterino. Revista de Ciencias Médicas. 2014;18(5): 753-66.

17. Rodríguez AM, Castillo MJ, Mejía W, Carmona DE, Marrero M, Acosta M. Relación del Virus del Papiloma Humano (HPV) con patologías del cuello uterino en la clínica de patologías del cérvix del Hospital Dr. Luis E. Aybar. Rev Med Dom. 2006;67(2): 130-2.

18. Franco Argote O, Valladares Vallín J, Pérez Ramos G. Neoplasia intraepitelial cervical en un área de salud. Rev Cubana Obstet Ginecol. 2011;37(2): 193-203.

19. Martínez Camilo V, Torrientes Hernández B. Neoplasia Cervical en la Mujer Climatérica. Rev Cubana Obstet Ginecol [en línea] 2011 [fecha de acceso 10 de marzo de 2015]; 37(2). Disponible en: http://scielo.sld.cu/scielo.php? script=sci_arttext\&lng=pt\&nrm=iso\&tlng=pt\& pid=S0138-600X2006000100001

20. Mhaske M, Jawadekar SJ, Saundale SG. Study of Association of Some Risk Factors \& Cervical Dysplasia / Cancer among Rural Women. National Journal of Community Medicine. 2011;2(2): 209-12. 